\title{
To Compare and Evaluate the Sealing Ability of Root Canal Sealer with and without Triple Antibiotic Paste using Ultraviolet-visible Spectrophotometric Analysis
}

\author{
${ }^{1}$ Deepa Deepak Shori, ${ }^{2}$ Pratima Ramakrishna Shenoi, ${ }^{3}$ Sonal Pradeep Dhote, ${ }^{4}$ Chetana Sachin Makade \\ ${ }^{5}$ Mohit Kumar Gunwal, ${ }^{6}$ Ameya Vasudeo Paralikar
}

\section{ABSTRACT}

Purpose: The aim of current study was to compare and evaluate the sealing ability of root canal sealer with and without triple antibiotic paste.

\begin{abstract}
Materials and methods: Fifty extracted human single rooted teeth were selected and each test group had 25 teeth. The teeth were decoronated and then prepared with protaper rotary files till the no $\mathrm{F} 2$ in conjunction with $17 \%$ EDTA lubrication and $2 \mathrm{ml}$ of $5.25 \%$ sodium hypochlorite irrigation. The teeth in group I were obturated using the zinc oxide sealer mixed with triple antibiotic paste and the teeth in group II were obturated using the zinc oxide sealer alone and then nail varnish was applied leaving the apical $3 \mathrm{~mm}$ of root exposed. The apical third was then immersed in $5 \mathrm{ml}$ of $2 \%$ methylene blue dye in $15 \mathrm{ml}$ screw capped bottle for 72 hours. The varnish was removed and the teeth were subsequently immersed in $35 \%$ nitric acid and kept again for 72 hours in a centrifugal tube. After the teeth were completely dissolved the solution was then filtered with a fine grit filter paper and centrifuged at $2000 \mathrm{rpm}$ for 1 minute. The collected solution was then subjected to spectrophotometric analysis. The data was evaluated statistically by Mann-Whitney U-test.
\end{abstract}

Results: The results showed significant differences $(p<0.05)$ between the sealing ability of two groups evaluated.

Conclusion: Present study suggests that addition of triple antibiotic paste to the sealer increased its sealing ability, reduced the microleakage and also imparts the antimicrobial property to the sealer.

Keywords: Triple antibiotic paste, Spectrophotometer, Amoxicillin, Ofloxacin, Ornidazole.

How to cite this article: Shori DD, Shenoi PR, Dhote SP, Makade CS, Gunwal MK, Paralikar AV. To Compare and EvaIuate the Sealing Ability of Root Canal Sealer with and without Triple Antibiotic Paste using Ultraviolet-visible Spectrophotometric Analysis. Int J Prosthodont Restor Dent 2014;4(2):48-51.

\footnotetext{
${ }^{1}$ Professor/Guide, ${ }^{2} \mathrm{Head},{ }^{3,6}$ Postgraduate Student

${ }^{4}$ Associate Professor, ${ }^{5}$ Senior Lecturer

${ }^{1-6}$ Department of Conservative Dentistry and Endodontics VSPM Dental College and Research Center, Nagpur, Maharashtra, India
}

Corresponding Author: Deepa Deepak Shori, Professor/Guide Department of Conservative Dentistry and Endodontics, VSPM Dental College and Research Center, Nagpur, Maharashtra, India Phone: 9422803637, e-mail:dr_sonal_dhote@yahoo.co.in
Source of support: Nil

Conflict of interest: None declared

\section{INTRODUCTION}

Successful endodontic treatment depends upon the complete elimination of polymicrobial pulpal pathogens which are Gram-positive and Gram-negative anaerobes and obtaining a three dimensional fluid tight seal at the apical foramina and accessory canals. ${ }^{1}$ Biomechanical preparation and proper irrigation alone are not sufficient to eliminate bacteria and provide sterile environment which will accelerate healing. In contrast to the vital pulp which is free of microorganisms a non-vital pulp is colonized by microbial ecosystem predominated by anaerobic growth. The recent theory of sterilization and repair suggest the use of triple antibiotic paste to reduce root canal pathogens. ${ }^{2}$ The root filling materials with antibacterial property can kill the bacteria and its communication with the periradicular region. ${ }^{3}$

Topical antibiotics may have an antibacterial effect limited in the pulp chamber. Systemic administration of antibiotic are adjuncts with endodontic procedure, but also carry the risk of side effects, such as allergies and resistance development. Grossman in 1957 prepared polyantibiotic paste containing penicillin, bactericin, streptomycin and caprylate sodium and used it as a successful intracanal medicament. ${ }^{4}$

Hoelscher studied that addition of polyantibiotics to the sealer increased the antimicrobial reaction of sealer to Enterococcus fecalis. ${ }^{1}$ No evidence of literature specifies the evaluation of the sealing ability of sealers in combination with antibiotics. So, the aim of present study was to compare and evaluate the sealing ability of commonly used zinc oxide sealer with and without antibiotics.

\section{MATERIALS AND METHODS}

Fifty freshly extracted single rooted human teeth were collected and stored in PBSC (Penicillin, bacitracin streptomycin and caprylate sodium) saline solution during all stages of study. X-rays were taken to confirm the presence of single canal. All the teeth included in the study were free from any caries, open apices, cracks, curved roots and resorp- 


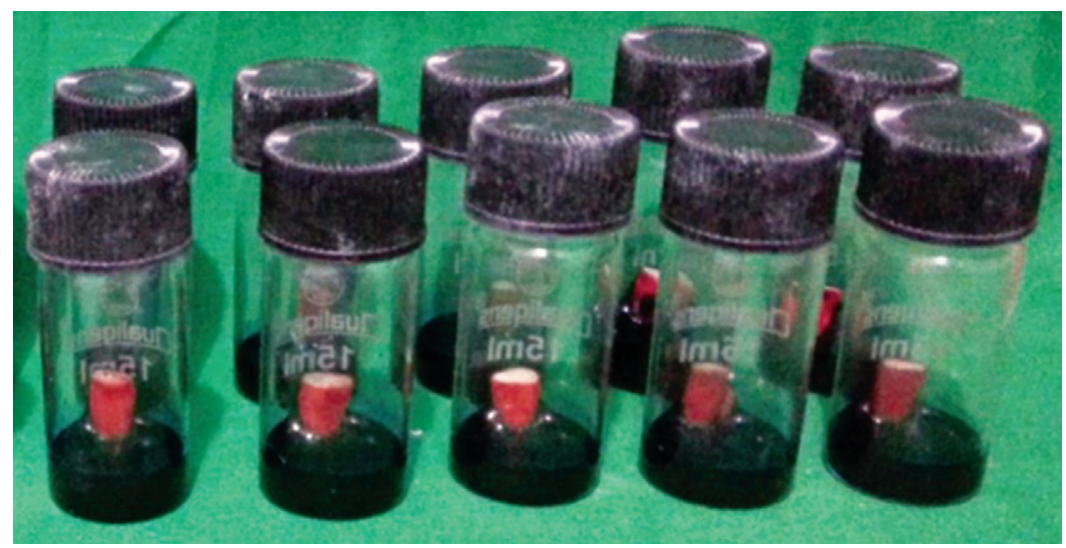

Fig. 1: Samples dipped in methylene blue dye

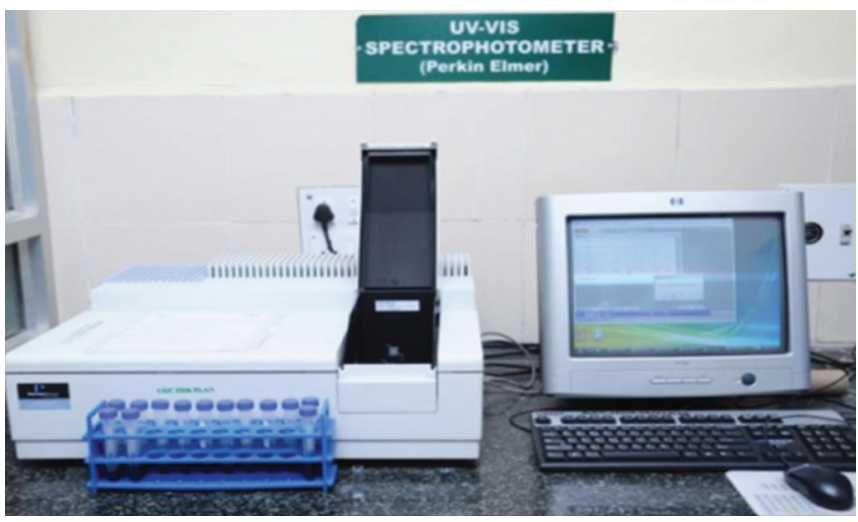

Fig. 2: Spectrophotometric analysis

tive defects. Proper cleaning of teeth was done. The teeth were decoronated at the cementoenamel junction by using diamond disks. The canal length was determined by using a size 10 K-type file (Kendo, Germany) and the working length was established by reducing $1 \mathrm{~mm}$ from the obtained length. The root canals were prepared with the rotary protaper files till F2 (Dentsply, Mallifer, Switzerland) with the crowndown technique until working length was reached. During the biomechanical preparation the canal patency was maintained with a size $10 \mathrm{~K}$-file and irrigation with 5.25\% sodium hypochlorite solution was carried out. After using the last file, the roots were irrigated with saline solution and dried with absorbant paper points (Dentsply, Germany). The canals were irrigated in between instrumentation with $17 \%$ ethylene diamine tetra-acetic acid (EDTA, Prime dental, India) and 5.25\% sodium hypochlorite.

This protocol was employed for all the samples used in the study. Finally the canals were irrigated with EDTA and saline and the debrided root canals were dried with multiple paper points and randomly divided into two experimental groups (25 for each group). Group I teeth were obturated with lateral condensation using triple antibiotic paste mixed with sealer system and the root canal opening was sealed with a temporary filling material (Cavit 3M ESPE, US). Triple antibiotic paste used in this study was prepared using amoxycilin, ofloxacin and ornidazole (Imox, IPCA laboratories; Ofloren $\mathrm{Oz}$, Indoco, India). The sugar coatings of the tablets was removed by just scraping of the outer surface and were then grounded into a fine powder using mortar and pestle. The powdered antibiotic and the sealer were then weighed on a digital weighing machine in a ratio of $1: 1$, and this combination of sealer + antibiotics was then used to obturate the group I. The teeth in group II were obturated using Gutta percha master point (Diadent International, Korea) dipped in zinc oxide sealer (Dentsply, Germany). Excess Gutta percha was removed and condensed with plugger; the root canal opening was sealed with a readymade temporary filling material (Cavit 3M ESPE, US).

\section{SAMPLE PREPARATION}

Samples from all the groups were coated with 2 coats of nail varnish except for the apical $3 \mathrm{~mm}$. After application of nail varnish, the samples were allowed to air dry. Apical third of samples which was left uncoated was then immersed in $5 \mathrm{ml}$ of $2 \%$ methylene blue dye solution in $15 \mathrm{ml}$ screw capped bottle (Fig. 1) and stored at $37^{\circ} \mathrm{C} \pm 2{ }^{\circ} \mathrm{C}$, at relative humidity in incubator for 72 hours. After that the samples were washed under running tap water to remove the traces of the dye and the nail varnish was removed using ultrasonic scalers. The teeth were then cleaned and immersed and stored in freshly prepared 35\% nitric acid for 72 hours in the centrifugal tube. After the teeth were completely dissolved the solution was filtered using fine grit filter paper into another centrifugal tube. The obtained sample solutions were finally centrifuged at $2000 \mathrm{rpm}$ for 1 minute. The supernatent solutions thus collected were subjected to spectrophotometric analysis (Fig. 2) using a filter of $670 \mathrm{~nm}$. The results were recorded as a measure of transmission of light (i.e. more light transmission suggestive of less microleakage). ${ }^{5}$

\section{RESULTS}

The result was recorded as a measure of transmission of light. According to Lambert-Beer's law, the magnitude of 
Table 1: Comparison of apical leakage between two groups, i.e. with and without triple antibiotic paste

\begin{tabular}{lllllll}
\hline Group & Mean & Median & $S D$ & Min. & Max. & $t$-value \\
\hline I & 84.4 & 87 & 6.80 & 69 & 92 & 5.4490 \\
II & 71.4 & 69 & 9.75 & 60 & 90 & - \\
\hline
\end{tabular}

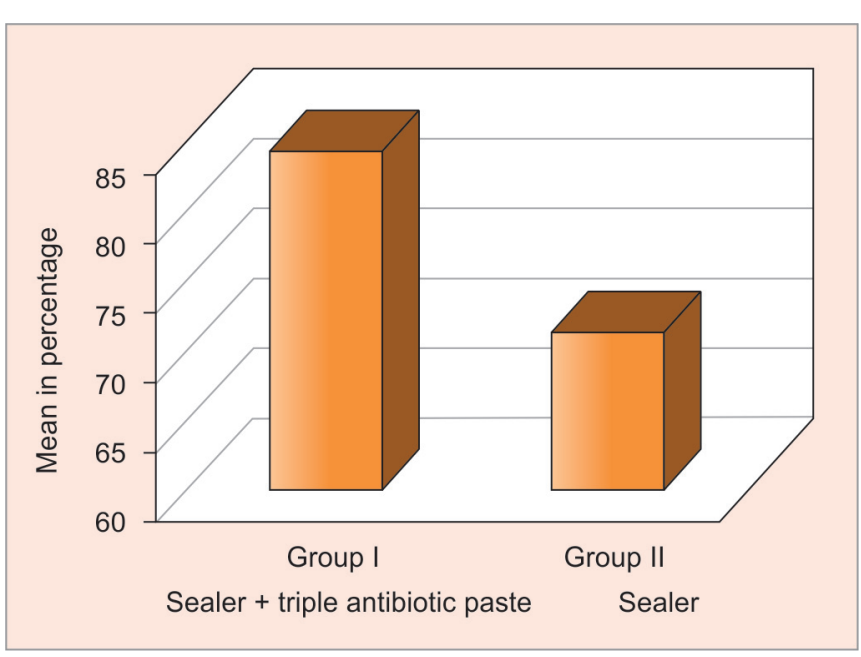

Graph 1: Comparison between two groups with and without triple antibiotic paste

absorbance is directly proportional to the concentration of dye in the solution and inversely proportional to the percentage of light transmission.

Thus, if the value of $\%$ of light transmission is less then it shows that the amount of dye penetrated in the samples is more.

Transmission values for each sample in both the groups were compared and analyzed using Mann-Whitney U-test. Apical leakage values were presented as mean \pm standard deviation. Median and range were also calculated. For normally distributed data, 2 sample independent t-test was done. $p$-value $<0.05$ was considered as statistically significant. Statistical software STATA version 12.0 was used for data analysis.

As shown in Table 1, the p-value is greater than 0.0001 which shows the highly significant difference between the two groups and Graph 1 shows less mean apical leakage with the use of sealer and triple antibiotic paste, i.e. more of light transmission in that group.

\section{DISCUSSION}

It has been reported that sterilization of root canal and periradicular lesion during endodontic treatment results in good healing of periapical lesion (endo and traumat 1987). Root canal infections are mostly polymicrobial in nature, so use of a single antibiotic to disinfect the canal is not feasible. ${ }^{2}$ During endodontic treatment antibiotics may be applied systemically or locally. However, it has been found that the local application of antibiotics within the root canal system may be a more effective mode than systemic application, owing to the adverse effects observed after systemic administration (Gilad et al 1999). ${ }^{4}$

The first local use of antibiotics was done by Grossman in 1951 where he used a poly antibiotic paste PBSC (penicillin, bacitracin, streptomycin and caprylate sodium). Later Weine (2003) replaced caprylate sodium with nystatin in similar medicament known as PBSN. ${ }^{4}$ Minimum inhibitory concentration (MIC) method used to determine the sensitivity of bacteria cannot alone determine the MIC for all species of bacteria and it may also not determine that combination of drugs can kill bacteria. ${ }^{6}$ Kreth et al (2008) studied that addition of silver ions to the endodontic sealer increases its antimicrobial activity against Streptococcus mutans. ${ }^{1}$ Ozan et al (2005) reported that healing in case of a large cyst like periradicular lesions gave successful results when a combination of antibiotic drugs was used as a dressing. ${ }^{7}$ Triple antibiotic paste was also found to be most effective in elimination of the bacteria in biofilms on intraorally infected dentin compared to $2 \%$ chlorhexidine gel and calcium hydroxide. ${ }^{8}$ Drugs, such as metronidazole even at a concentration of $100 \mu \mathrm{g} \mathrm{ml}^{-1}$ could not kill all the bacteria from carious lesion (Hoshino et al 1980). ${ }^{9}$ So, some additional drug was added to sterilize the lesion and it was found that mixture of antibacterial drugs (metronidazole + ciprofloxacin + minocycline) could sterilize the carious lesion, necrotic pulp and even the infected root dentin (Sato et al 1992). ${ }^{9}$

In the present study, ornidazole has been used as it has a wide spectrum of bactericidal action against oral anaerobes and also against infected necrotic pulp. This drug was combined with ofloxacin and amoxicillin to form a triple antibiotic paste. It has been found that during the local application if a combination drug is used, a very low dose is required, so also the side effects of drugs are minimized. Drugs, such as minocycline are found to cause pigmentation, hence amoxicillin has been used in the study. Reynolds et al suggested the use of root canal projector for the placement of triple antibiotic paste to prevent any type of coronal discoloration. ${ }^{10}$ Takushige $\mathrm{T}$ et al stated that the drugs can be powdered and mixed with either macrogolpropylene glycol or a canal sealer. $^{2}$

Till date many studies were conducted only on the antibacterial efficacy of antibiotic combinations used. So, this study concentrates on the sealing ability of the sealer combined with triple antibiotic paste. Various methods are used to check the apical sealing ability, such as dye pene- 
tration, dye extraction, bacterial penetration, radioisotope penetration and fluid infiltration techniques. The technique used here is dye extraction method where the teeth were completely dissolved in acid, liberating all the dye from within, hence making possible to measure the total amount of dye penetrated.

Ultraviolet-visible spectrophotometric analysis of dye extraction is easy and have minimal human measurement errors and provides determination of volumes of leakage, rather than linear measurements as reported by Sangappa V et al (2005) and Meena Kumari C.

From the results of the present study, it can be concluded that addition of triple antibiotic paste (amoxycillin, ofloxacin and ornidazole) to the zinc oxide sealer increased the antibacterial effect of the sealer. In cases with pulpal necrosis and apical periodontitis sealer with high antimicrobial activity should be used to avoid the growth of remaining microorganisms. ${ }^{11}$ The zinc oxide based sealer are found to have antibacterial effect due to the presence of eugenol which is an important antibacterial agent. ${ }^{12}$ This sealer improved the postobturation apical seal which is an important factor in endodontic treatment and thus decreasing the apical microleakage. Also the results of this study coincide with the other study (Sandhya S et al 2013) and it can be said that the antibiotic additives added may have increased the density of the combination, i.e. antibiotic + sealer as compared to sealer alone. ${ }^{1}$

Other few advantages of using triple antibiotic paste (TAP) are, some bacterial strains are resistant to some specific antibiotics, hence combination of drugs is more effective $^{2}$ and also TAP is useful for sterilization of lesions and tissue repair. ${ }^{2}$ TAP can promote functional development of pulp dentin complex and also useful in endodontic regenerative procedure. ${ }^{13}$ Use of TAP as an intracanal medicament after the irrigation results in statistical reduction of bacteria. ${ }^{13}$ Most important is that it is biocompatible. Few disadvantages also to be taken in considerations such, as some of the drugs like minocycline can cause discoloration of tooth so its use should be limited to the canals only. It takes a little time to scrape the sugar coatings and to prepare TAP as it is not available on routine basis. ${ }^{13}$

Use of ultraviolet-visible spectrophotometric analysis is more advantageous as it is simple, rapid, moderately specific and applicable to small quantities of compounds. Also it is one of the most frequently employed technique in pharmaceutical analysis. It involves measuring the amount of ultraviolet or visible radiation absorbed by a substance in solution. ${ }^{14}$ More comparative studies need to be carried out along with ultraviolet-visible spectrophotometer to rule out the errors and other disadvantages.
In the above study, only three antibiotics were used and also the sealer + antibiotic paste was kept for 72 hours, so the further research studies can be carried out with other combination of drugs and with extended period of time.

\section{CONCLUSION}

Addition of three different antibiotics (triple antibiotic paste) to the sealer was found to increase its thickness and density thus improving its sealing property. It was also found to be effective in reducing the apical microleakage and also imparts the antimicrobial property to the sealer.

\section{REFERENCES}

1. Shrestha S, Kundabala M. Evaluation of sealing ability of root canal sealer with various antibiotic additives: An in vitro study. J Interdisciplinary Dentist 2013;3:21-24.

2. Varalaxmi RP, Banker SM. 3 Mix-MP in endodontics: an overview. JDMS 2012;3:36-45.

3. Ingle JI, Bakland LK. Endodontics. 5th ed. Hamilton, Ontario, India: BC Decker, elsevier 2006.p1009.

4. Mohammadi Z, Abbot PV. On the local applications of antibiotics and antibiotic-based agents in endodontics and dental traumatology. Int Endo J 2009;42:555-567.

5. Available at: www.ce.udel.edu/ imhoff/.../file.../Spectrophotometry_handouts. pdf. Assessed on 1st November 2013.

6. William W, Fabricio T, Linda L, Asgeir S, Martin T. Disinfection of immature teeth with a triple antibiotic paste. J Endod 2005;31:439-443.

7. Ozan, Kursat ER. Endodontic treatment of a large cyst-like periradicular lesion using a combination of antibiotic drugs: a case report. J Endod 2005;31:898-900.

8. Ronald OZ, Clovis MB, Paloma GM, Bruno C, et al. Antimicrobial activity of triantibiotic paste, $2 \%$ chlorhexidine gel and calcium hydroxide on an intraoral-infected dentin biofilm model.

9. Hoshino E, Khurana-Ando N, Sato, Uematsu H, Sato M, Kota $\mathrm{K}$, Iwaku M. In vitro antibacterial susceptibility of bacteria taken from infected root dentine to a mixture of ciprofloxacin, metronidazole and minocycline. Int Endod J 1996;29: 125-130.

10. Kim JH, Kim Y, Shin SJ. Tooth discolouration of immature permanent incisor associated with triple antibiotic therapy: a case report. Endod 2010;36:1086-1091.

11. Funda KC, Halil CA, Osman E. In vitro antibacterial activities of root canal sealers by using two different methods. J Endod 2004;30:57-60.

12. Anita AH, James KB, James SM. In vitro evaluation of the antibacterial effects of a root canal sealer antibiotic combination against Enterococcus faecalis. JOE 2006;32:145-147.

13. Vijayaraghavan R, Mathian VM, Sundaram MA, Karunakaran $\mathrm{R}$, Vinodh S. Triple antibiotic paste in root canal therapy. J Pharm Bioallied Sci 2012 Aug;4(Suppl 2):S230-S233.

14. Siladitya B, Subhajit G, Fahad A, Saayak S, Sritoma B. UVvisible spectrophotometric method development and validation of assay of paracetamol tablet formulation. J Anal Bioanal Techniques 2012;3:6. 